Draft Version November 4, 2018

Preprint typeset using $\mathrm{LAT}_{\mathrm{E}} \mathrm{X}$ style emulateapj v. 08/22/09

\title{
MAGNETIC FIELD AMPLIFICATION AND RAPID TIME VARIATIONS IN SNR RX J1713.7-3946
}

\author{
Donald C. Ellison ${ }^{1}$ AND Andrey Vladimirov ${ }^{1}$ \\ Draft version November 4, 2018
}

\begin{abstract}
Evidence is accumulating suggesting that collisionless shocks in supernova remnants (SNRs) can amplify the interstellar magnetic field to hundreds of microgauss or even milli-gauss levels, as recently claimed for SNR RX J1713.7-3946. If these fields exist, they are almost certainly created by magnetic field amplification (MFA) associated with the efficient production of cosmic rays by diffusive shock acceleration (DSA) and their existence strengthens the case for SNRs being the primary source of galactic cosmic ray ions to the 'knee' and beyond. However, the high magnetic field values in SNRs are obtained exclusively from the interpretation of observations of radiation from relativistic electrons and if MFA via nonlinear DSA produces these fields the magnetic field that determines the maximum ion energy will be substantially less than the field that determines the maximum electron energy. We use results of a steady-state Monte Carlo simulation to show how nonlinear effects from efficient cosmic ray production and MFA reduce the maximum energy of protons relative to what would be expected from test-particle acceleration.

Subject headings: acceleration of particles, shock waves, cosmic rays, supernova remnants, magnetic field, maximal energy
\end{abstract}

\section{INTRODUCTION}

Magnetic fields with strengths well above what can be expected from shock compression of the interstellar medium (ISM) field, $B_{\mathrm{ISM}}$, are inferred in supernova remnants (SNRs) from four types of observations: (1) sharp X-ray edges or filaments (e.g., Bamba et al. 2003; Warren et al. 2005; Cassam-Chenaï et al. 2007), (2) broadband fits of emission from radio to $\gamma$-ray energies (e.g., Cowsik \& Sarkar 1980; Vink \& Laming 2003; Völk, Berezhko \& Ksenofontov 2005), (3) spectral curvature in radio emission (Revnolds \& Ellison 1992) and, (4) with the recently reported Chandra observations of bright filaments in SNR RX J1713.7-3946 (Uchivama et al. 2007), rapid time variations of nonthermal X-ray emission. The most likely explanation for these fields is that $B_{\mathrm{ISM}}$ is amplified as part of the efficient production of cosmic rays by nonlinear diffusive shock acceleration (DSA) (e.g., Bell \& Lucek 2001; Amato \& Blasi 2006; Vladimirov, Ellison \& Bykov 2006). Since the magnetic field largely determines the maximum particle energy a shock can produce, magnetic field amplification (MFA) is critical for interpreting $\mathrm{GeV}$ $\mathrm{TeV}$ emission and determining if SNRs produce cosmic rays to the 'knee' and above. Equally important is the fact that if strong MFA is an intrinsic part of nonlinear DSA, unexpectedly strong magnetic fields may be present in other systems undergoing efficient shock acceleration such as radio jets and gamma-ray burst (GRB) afterglows. The detailed modeling of broadband observations of SNRs is the only practical way to determine the importance of nonlinear effects and MFA in high Mach number collisionless shocks.

Ironically, the evidence for large magnetic fields and, therefore, nonlinear MFA is obtained exclusively from ra-

\footnotetext{
${ }^{1}$ Physics Department, North Carolina State University, Box 8202, Raleigh, NC 27695, U.S.A.; don_ellison@ncsu.edu, avladim@ncsu.edu
}

diation emitted by relativistic electrons, while the nonlinear processes responsible for MFA are driven by the efficient acceleration of relativistic ions, mainly protons. Relativistic protons can only be directly inferred from pion-decay emission at $\mathrm{GeV}-\mathrm{TeV}$ energies and the unambiguous interpretation of these observations is difficult and remains uncertain. In any case, even if pion-decay emission is clearly present, these observations must be combined with synchrotron emission from electrons to estimate the magnetic field strength.

A recent report by Uchivama et al. (2007) of rapid time variations $(\sim 1 \mathrm{yr})$ in the nonthermal X-ray emission from bright filaments in SNR RX J1713.7-3946 provides new clues to the electron acceleration process in SNRs. As emphasized by Uchivama et al. (2007), the rapid time variations suggest two conclusions. First, if the variations are determined by the synchrotron radiation losses of $\mathrm{TeV}$ electrons, the magnetic field can indeed be amplified to milligauss-levels at a SNR shock and, second, the MFA process is tightly bound with the production of relativistic particles (i.e., CRs). An important consequence of these conclusions is that the fraction of the shock ram kinetic energy that is put into accelerated ions must be large, e.g., tens of percent. Therefore, DSA must be in an efficient, nonlinear regime where the feedback of accelerated particles on the shock structure is significant. A simple estimate confirming this consequence is equation (15) in Bell \& Lucek (2001).

Here we address a single question: Can, as asserted by Uchiyama et al. (2007), the large amplified fields inferred for electrons from radiation losses in a nonlinear shock also determine the maximum proton energy produced in the SNR shock? We find the answer to be no because the inevitable nonlinear shock modification (due to efficient DSA) and the magnetic field variation in the shock precursor (due to MFA) make the maximum proton energy smaller than what is expected without accounting for these effects. Using results from 
Vladimirov, Ellison \& Bykov (2006) for DSA limited by the finite size of the shock, we estimate that the maximum proton energy is at least an order of magnitude less than that predicted assuming an unmodified shock with a large, nearly constant field inferred from electron radiation losses. Our result is similar to that found by Blasi. Amato \& Caprioli (2007) in a time-dependent calculation of DSA where the acceleration is limited by the age of the shock rather than the size, an indication that the nonlinear effects we discuss are robust.

\section{ACCELERATION IN A CR MODIFIED SHOCK}

The commonly accepted picture of nonlinear DSA is the following: accelerated protons diffuse upstream of the shock front, carrying a significant fraction of the shock ram kinetic energy, and 'push' on the incoming plasma. In order to conserve momentum, the shock forms a smooth precursor where the flow speed (in the shock frame) gradually decreases from the far upstream speed, $u_{0}$, to a pre-subshock speed, $u_{1}$, and then sharply decreases in a viscous subshock to the downstream speed, $u_{2}$. Here, $R_{\text {tot }}=u_{0} / u_{2}$ is the overall compression ratio and $R_{\text {sub }}=u_{1} / u_{2}$ is the subshock compression ratio. If MFA accompanies this nonlinear shock smoothing, the low ISM field is amplified from $B_{0}=B_{\text {ISM }}$ to $B_{2}$ on the same precursor length scale. ${ }^{2}$

If electrons suffer strong radiation losses, they will have a lower maximum energy $E_{e}^{\max }$ than protons and will remain in the vicinity of the subshock, spending most of their time downstream as they radiate in the strongly amplified downstream field $B_{2}$. Protons, on the contrary, sample the whole precursor and experience a weaker mean field $\langle B(x)\rangle$, which is between $B_{\text {ISM }}$ and $B_{2}$. Because the escape of protons from the shock occurs far upstream, where the magnetic field is low, one can expect that for the most energetic protons, $\langle B(x)\rangle$ is closer to the weak $B_{\text {ISM }}$ than to the strong $B_{2}$, and, consequently, their maximum energy $E_{p}^{\max }$ is lower than would be estimated assuming that the shock is unmodified with $B(x) \sim B_{2}$ everywhere. The latter case, $B(x) \sim B_{2}$ everywhere, is implicitly assumed by Uchivama et al. (2007) when they write $t_{\mathrm{acc}}=0.1(B / \mathrm{mG})^{-1}(E / \mathrm{TeV}) \mathrm{yr}$ for the proton acceleration time.

In a size limited shock, the proton maximum energy, $E_{p}^{\max }$, will be determined when the upstream diffusion length of the most energetic protons becomes comparable to the confinement size of the shock, typically some fraction of the shock radius. We model the confinement size with a free escape boundary (FEB) at a distance $L_{\mathrm{FEB}}$ in front of the shock. Protons that reach this position stream freely away from the shock without producing any more magnetic turbulence. Therefore:

$$
L_{\mathrm{FEB}} \sim D\left(E_{p}^{\max }\right) / u_{\mathrm{sk}},
$$

where for the diffusion coefficient, $D(E)$, we assume

$$
D(E)=\lambda c / 3=\eta r_{g} c / 3,
$$

and $u_{\text {sk }}$ is the upstream flow speed, $\lambda$ is the scattering mean free path, $r_{g}$ is the gyroradius, and $\eta$ is a parameter that characterizes the scattering strength. None of

\footnotetext{
2 Here and elsewhere the subscript " 0 " implies far upstream from the shock and "2" implies downstream.
}

our conclusions depend importantly on $\eta$ and we assume 'Bohm' diffusion and set $\eta=1$. Since for ultrarelativistic protons $r_{g}=E /(e B)$, equations (1) and (2) determine $E_{p}^{\max }$ for a given $L_{\mathrm{FEB}}$, i.e.,

$$
E_{p}^{\max } \propto L_{\mathrm{FEB}} u_{\mathrm{sk}} B_{\mathrm{sk}} .
$$

For a quasi-parallel, unmodified (UM) shock with no MFA, the maximum proton energy $\left.E_{p}^{\max }\right|_{\mathrm{UM}}$ is thus defined by (3) with $u_{\mathrm{sk}} B_{\mathrm{sk}}=u_{0} B_{0}=u_{0} B_{2}, u_{0}$ being the shock speed and $B_{2}$ being the downstream magnetic field derivable from synchrotron emission of accelerated electrons. However, for a nonlinear (NL) CR modified shock of the same physical confinement size, $L_{\mathrm{FEB}}$, the maximum proton energy $\left.E_{p}^{\max }\right|_{\mathrm{NL}}$ will be determined by some mean value $\langle u(x) B(x)\rangle$, giving

$$
\frac{\left.E_{p}^{\max }\right|_{\mathrm{NL}}}{\left.E_{p}^{\max }\right|_{\mathrm{UM}}}=\frac{\langle u(x) B(x)\rangle}{u_{0} B_{2}} .
$$

For a strongly modified shock, $\langle u(x) B(x)\rangle \ll u_{0} B_{2}$, and in the following we determine $\langle u(x) B(x)\rangle /\left(u_{0} B_{2}\right)$ using the Monte Carlo model described in detail in Vladimirov, Ellison \& Bykov (2006).

\section{MONTE CARLO SIMULATION}

The Monte Carlo model we use (see Vladimirov, Ellison \& Bykov 2006, for full details) calculates NL DSA and the magnetic turbulence produced in a steady-state, plane-parallel shock precursor by the CR streaming instability. While the quasi-linear approximations we make are only valid in the linear regime we nevertheless follow Bell \& Lucek (2001) and assume that the instability doesn't saturate or damp at $\Delta B \approx B_{0}$ but continues in the nonlinear regime with $\Delta B \gg B_{0}$. With this approximation, we are able to self-consistently determine the nonlinear shock structure [i.e., $u(x)$ vs. $x]$, the MFA $\left[B_{\text {eff }}(x)\right.$ vs. $\left.x\right]$, and the thermal particle injection. ${ }^{3}$

The NL results we investigate do not depend qualitatively on the particular shock parameters as long as the sonic Mach number is large enough to result in efficient DSA. Here, we use a shock speed $=u_{0}=3000 \mathrm{~km} \mathrm{~s}^{-1}$, sonic Mach number $M_{s} \approx 30$, plasma density $n_{\mathrm{ISM}}=1$ protons $\mathrm{cm}^{-3}$, and $B_{0}=B_{\mathrm{ISM}}=10 \mu \mathrm{G}$, yielding an Alfvén Mach number, $M_{A} \approx 140$. To these parameters we add a FEB boundary at $L_{\mathrm{FEB}} \sim 0.1 \mathrm{pc}$, corresponding to $10^{8} r_{g, 0}$, where $r_{g, 0} \equiv m_{p} u_{0} c /\left(e B_{0}\right)$. This size is comparable to the hot spots in SNR RX J1713.7-3946 and produces a proton energy $\sim 10^{15} \mathrm{eV}$ in our unmodified shock approximation.

Using the above parameters, we simulate two cases: a nonlinear solution, where $B$ is amplified from an upstream value $B_{0}=10 \mu \mathrm{G}$ to a downstream value $B_{2}=$ $450 \mu \mathrm{G}$ (obtained self-consistently by our model), and an unmodified solution with a magnetic field set equal everywhere to $B_{2}=450 \mu \mathrm{G}$. In these two cases we look at $E_{p}^{\max }$ to see how the prediction of the NL model, conserving momentum and energy, compares to the prediction

3 Note that the Monte Carlo model ignores the dynamic effects of electrons and the NL shock structure is determined solely from the backpressure of protons and the magnetic field. While electron acceleration can be modeled (e.g., Baring et al.1999), we only show proton spectra here. 


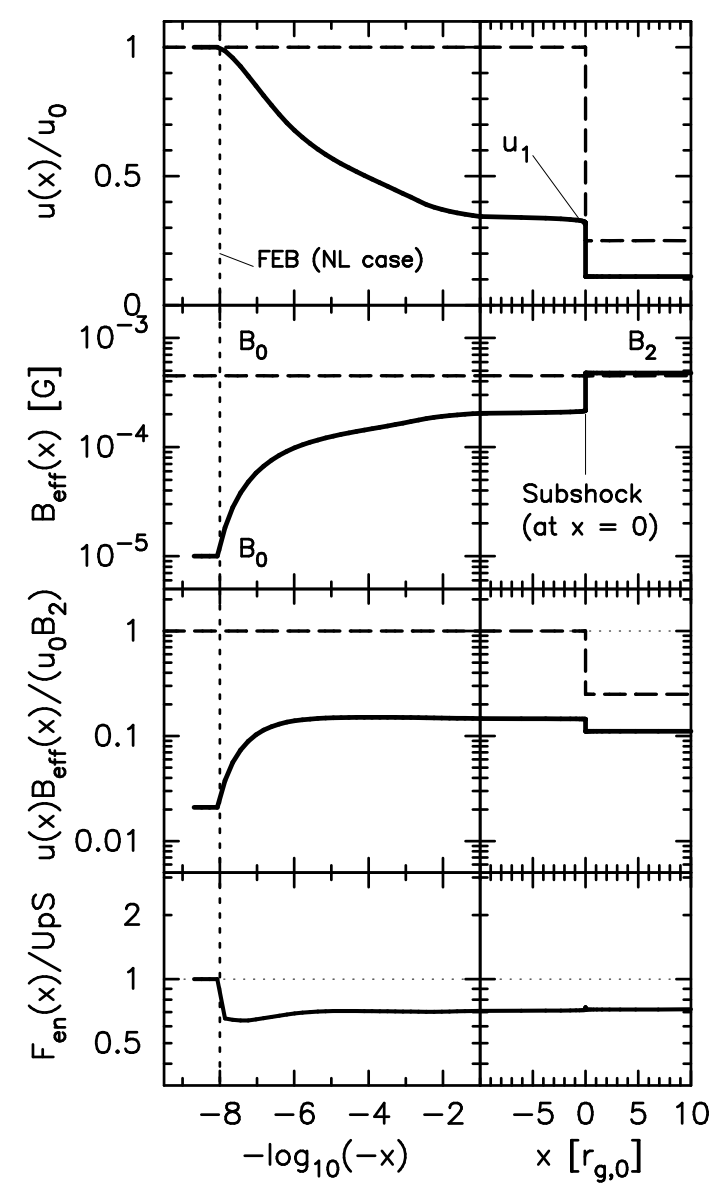

FIG. 1.- As labeled, the panels show the bulk flow speed in units of the shock speed, $u_{0}$, the effective magnetic field, $B_{\text {eff }}$, $\left[u(x) B_{\text {eff }}(x)\right] /\left(u_{0} B_{2}\right)$, and the energy flux, $F_{\text {en }}(x)$, in units of the far upstream energy flux, all versus position, $x$. The subshock is at $x=0$ and $r_{g, 0} \equiv m_{p} u_{0} c /\left(e B_{0}\right)$ (note the change in $x$-scale between linear and $\log _{10}$ at $\left.x=-10 r_{g, 0}\right)$. In all panels, the solid curves are the self-consistent, NL result and the dashed curves are for an UM shock where energy and momentum are not conserved. For the UM shock, the $x$-independent $B_{\text {eff }}=B_{0}=B_{2} \simeq 450 \mu \mathrm{G}$ has been chosen to match the amplified field, $B_{2}$, in the NL result. Note that the $x$-scale in physical units depends on $B_{0}$ which is different in the two cases but that the FEB is set to the same physical distance in both cases, i.e., $L_{\mathrm{FEB}} \sim 0.1 \mathrm{pc}$. The drop in energy flux at the FEB in the bottom panel results as energetic protons escape the NL shock and carry away energy. The total energy flux is conserved in the NL case.

of the UM model, implicitly assumed by Uchivama et al. (2007). The information about the maximum energy of electrons (which are not included in our calculations) can be inferred graphically from the plot of the acceleration time (see Fig. 2).

Figure 1 shows the shock structure, $u(x)$, the effective magnetic field after amplification, $B_{\text {eff }}(x)$, and $u(x) B_{\text {eff }}(x) /\left(u_{0} B_{2}\right)$, for the unmodified case (dashed lines), and the nonlinear case (solid lines). The bottom panel shows the energy flux, normalized to the far upstream value, for the NL case. The smoothing of $u(x)$, the weak subshock $\left(R_{\mathrm{sub}} \simeq 2.9\right)$, and the increase in $R_{\mathrm{tot}}$ above $4\left(R_{\mathrm{tot}} \simeq 9\right)$ are clearly present in the top panel for the NL case. These three effects must occur to conserve momentum and energy if CRs are efficiently accelerated. The quantity $u(x) B_{\text {eff }}(x) /\left(u_{0} B_{2}\right) \sim 0.1$ over most of the precursor in the NL case.

In Figure 2 we show the momentum phase-space dis-

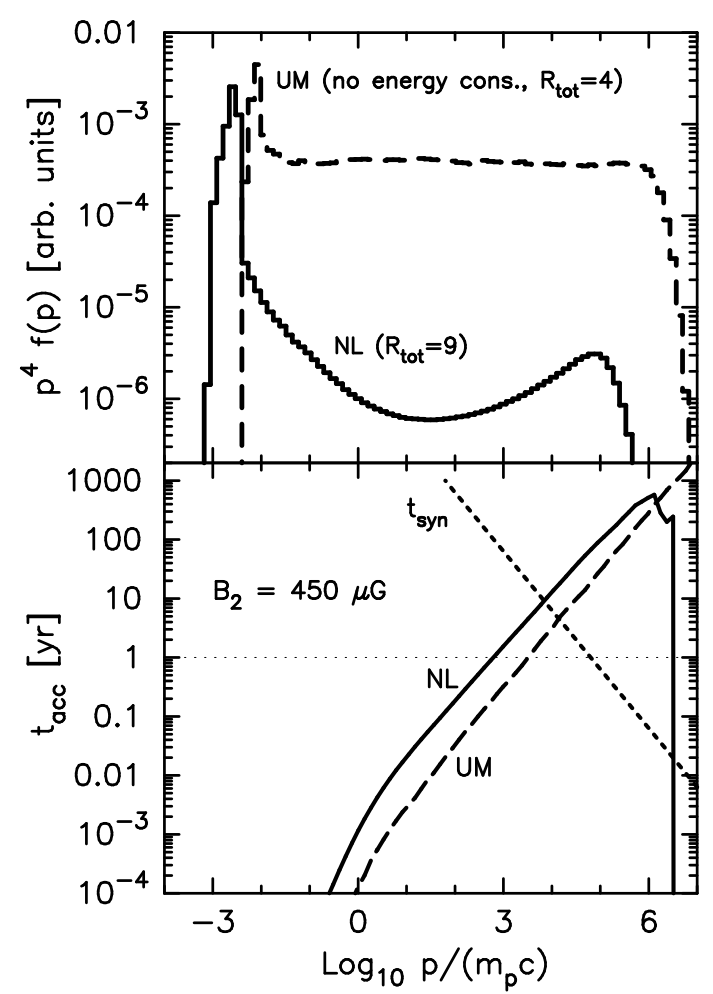

FIG. 2.- The top panel shows proton spectra, $p^{4} f(p)$, and the bottom panel shows the acceleration time to a given momentum for the shocks shown in Fig. 1] In both panels, the solid curve is the NL case and the dashed curve is the UM case. The maximum momentum in the UM case is about a factor of 10 larger than in the NL case. The dotted line in the bottom panel is the synchrotron loss time, $t_{\mathrm{syn}} \simeq 12(B / \mathrm{mG})^{-2}(E / \mathrm{TeV})^{-1} \mathrm{yr}$, for $B=0.45 \mathrm{mG}$. The NL shock accelerates particles at a slower rate than the UM shock.

tributions functions, $f(p)$ (multiplied by $p^{4}$ ), and the acceleration time, $t_{\mathrm{acc}}$. The NL effects evident in Fig. 1 result in,

$$
p_{\mathrm{NL}}^{\max } / p_{\mathrm{UM}}^{\max } \lesssim 0.1,
$$

and a longer $t_{\mathrm{acc}}$ to a given momentum. Here, $p_{\mathrm{NL}}^{\max }=$ $\left.E_{p}^{\max }\right|_{\mathrm{NL}} / c$ and $p_{\mathrm{UM}}^{\max }=\left.E_{p}^{\max }\right|_{\mathrm{UM}} / c$. We have not attempted a detailed fit to SNR RX J1713.7-3946, but note that the concave shape of our proton spectrum, above the thermal peak, is similar to that obtained by Berezhko \& Völk (2006) who find a good fit to the data, including the HESS TeV observations Aharonian et al. 2006).

The UM result is obtained from the Monte Carlo simulation assuming the same "thermal leakage" model for injection as in the NL result (e.g., Jones \& Ellison 1991). This injection scheme works self-consistently with modifications in the shock structure and overall compression ratio, $R_{\text {tot }}$, to conserve momentum and energy in the NL case. In the UM case, the shock structure and $R_{\text {tot }}$ are not adjusted and the thermal leakage model produces far too many injected particles to conserve momentum and energy. For the UM shock to become a test-particle shock with energy conservation, far fewer particles would need to be injected so that the normalization of the superthermal $f(p) \propto p^{-4}$ power law becomes low enough, relative to the thermal peak, so that in contains an insignificant fraction of the total shock ram kinetic energy. Since we are only interested in comparing $p_{p}^{\max }$ in the two 
cases, the normalization of the unmodified power law is unimportant since $p_{\mathrm{UM}}^{\max }$ only depends on $L_{\mathrm{FEB}}$.

\section{DISCUSSION AND CONCLUSIONS}

The possibility of strong MFA in SNR shocks has been strengthened by the recent observations of rapid time variability in hot spots in SNR RX J1713.7-3946 by Uchivama et al. (2007). If we accept the conclusions of Uchivama et al. (2007), the $\sim 1$ yr variations in X-ray emission in some hot spots stem from radiation losses for electrons and indicate magnetic fields on the order of $1 \mathrm{mG}$. Such large fields would almost certainly be caused by MFA occurring simultaneously with the efficient production of CR ions in DSA.

While a number of other interpretations of the X-ray and broadband emission in SNR RX J1713.7-3946 have concluded that the magnetic field present in the particle acceleration site is considerably less than $1 \mathrm{mG}$ (e.g., Ellison. Slane \& Gaensler 2001; Lazendic et al. 2004; Berezhko \& Völk 2006; Porter, Moskealenko \& Strong 2006), we have shown that even if the magnetic field inferred from electron radiation losses is as high as Uchivama et al. (2007) claim, the underlying physics of MFA in DSA shows that this field cannot be simply applied to protons to estimate their maximum energy.

The essential point is that, if MFA to milligauss levels is occurring as part of DSA, the acceleration must be efficient and the system is strongly nonlinear. The accelerated particles and the pressure from the amplified field feedback on the shock structure (Fig. 1) and this feedback makes the precursor less confining [i.e., $\left.\langle u(x) B(x)\rangle \ll u_{0} B_{2}\right]$. Therefore, a shock of a given physical size will not be able to accelerate protons to an energy as large as estimated ignoring NL effects. Even if the proton acceleration is limited by the finite age of the shock rather that the finite size, as we have assumed, the proton $E_{p}^{\max }$ will be less than in a TP approximation because the highest energy protons will have their acceleration time determined by $\langle u(x) B(x)\rangle$ rather than $u_{0} B_{2}$. This point has been made by Blasi. Amato \& Caprioli (2007) using a time-dependent, semi-analytic calculation of DSA with MFA. They have derived a general equation and determined $p_{\mathrm{NL}}^{\max }$ for a range of Mach numbers and choices for the diffusion coefficient when the acceleration is limited by the shock age. Considering the differences in the models and the specific parameters chosen for our examples of young SNRs, the fact that both calculations yield $p_{\mathrm{NL}}^{\max } / p_{\mathrm{UM}}^{\max } \lesssim 0.1$, indicates the robust nature of the result which should apply in any shock efficiently producing CRs and magnetic turbulence.

We note that we have neglected the potentially important physical effect of magnetic field damping in the strong downstream turbulence (e.g., Pohl, Yan \& Lazarian 2005). The importance of damping is still uncertain (see Cassam-Chenaï et al. 2007), but if it occurs it will be most important for high energy protons with long diffusion lengths. In effect, damping may determine the FEB but shouldn't qualitatively change the results for $p_{\mathrm{NL}}^{\max } / p_{\mathrm{UM}}^{\max }$.

Despite the reduction in $E_{p}^{\max }$ compared to testparticle predictions that our results imply, a remnant such as SNR RX J1713.7-3946 might still produce CRs up to the knee. The NL example we have presented with $B_{2} \simeq 450 \mu \mathrm{G}$ produces protons up to $\sim 100 \mathrm{TeV}$ in $\sim 100 \mathrm{yr}$ in a confinement region of $\sim 0.1 \mathrm{pc}$. If instead we had taken $L_{\mathrm{FEB}}=1 \mathrm{pc}$, a size comparable to the western shell of SNR RX J1713.7-3946, our NL model would produce $\sim 1 \mathrm{PeV}$ protons in $\sim 1000 \mathrm{yr}$. Protons of this energy are consistent with the $\sim 30 \mathrm{TeV} \gamma$-rays observed from SNR RX J1713.7-3946 (Aharonian et al. 2006) and when the acceleration of heavy ions such as $\mathrm{Fe}^{+26}$ is considered, the maximum particle energy extends to $>10^{16} \mathrm{eV}$.

The production of turbulence in efficient DSA is an active area of research and the question arises if non-resonant wave generation will substantially modify our results. For instance, recently Pelletier. Lemoine \& Marcowith (2006) have investigated Bell's non-resonant turbulence generation mechanism (Bell 2004) and found that it can dominate over resonant instabilities for high speed shocks (e.g., $u_{\text {sk }} \simeq$ $0.1 c)$. They also find, however, that for slower shocks of the order we assume here $\left(u_{\mathrm{sk}} \sim 0.01 c\right)$, resonant instabilities dominate. The Vladimirov, Ellison \& Bykov (2006) model assumes resonant instabilities.

Other effects may be important as well such as particles escaping at the FEB. In a size limited shock, the escaping energy flux can be large if DSA is efficient. For our example, the bottom panel of Fig. 2 shows that $\sim 30 \%$ of the energy flux escapes at the upstream FEB and this highly anisotropic beam of particles with energies near $E_{p}^{\max }$ moving through the cold ISM should efficiently generate turbulence. The details of this so-called "magnetic bootstrap" scenario (e.g., Blandford \& Funk 2007) are just starting to be investigated but such turbulence might generate higher effective fields with a correspondingly higher $E_{p}^{\max }$. Despite these possibilites and the uncertainties involved in wave generation, we believe none of these effects will qualitatively change our conclusions: the precursor field must still range from $B_{0}$ to $B_{2}$ and the proton maximum energy will be determined by a smaller effective field than that estimated from synchrotron losses for electrons.

As a final comment we emphasize a point also made by Blasi. Amato \& Caprioli (2007). If MFA is occurring and the system is highly NL, it may not be possible to explain temporal variations in nonthermal X-ray emission simply as a radiation loss time. There cannot be variations in X-ray emission on short time scales unless the accelerator changes in some fashion on these time scales, otherwise the radiation would be steady, or varying on the shock dynamic timescale, regardless of how short the radiation loss time was. Since the injection and acceleration of protons and electrons is nonlinearly connected to the amplified magnetic field, changes in the electron particle distribution and changes in the field producing the synchrotron emission, will go together and it may be difficult to unambiguously determine the field strength from temporal variations.

We thank R. Blandford, Y. Butt, and S. Funk for helpful discussions and acknowledge support from NASA grants ATP02-0042-0006, NNH04Zss001N-LTSA, and 06-ATP06-21. D.C.E. wishes to thank the Kavli Institute for Particle Astrophysics and Cosmology (KIPAC) where part of this work was done. 


\section{REFERENCES}

Aharonian, F. et al. 2006, A\&A, 449, 223.

Amato, E. \& Blasi, P. 2006, MNRAS, 371, 1251

Bamba, A., Yamazaki, R., Ueno, M. \& Koyama, K., 2003, ApJ, 589,827

Baring, M.G., Ellison, D.C., Reynolds, S.P., I. A. Grenier, I.A. \& Goret, P. 1999, ApJ, 513, 311

Bell, A. R. 2004, MNRAS, 353, 550

Bell, A. R. \& Lucek, S. G. 2001, MNRAS, 321, 433

Berezhko, E. G. and Völk, H. J. 2006, A\&A, 451, 981

Blandford, R. \& Funk, S. 2007, AIP Conf. Series, Eds. Ritz, S., Michelson, P. \& Meegan, C.A. 921, 62 (SLAC-PUB-128731)

Blasi, P., Amato, E. \& Caprioli, D., 2007, MNRAS, 375, 1471

Cassam-Chenaï, G., Hughes, J. P., Ballet, J. \& Decourchelle, A., 2007, ApJ, 665, 315

Cowsik, R. \& Sarkar, S., 1980, MNRAS, 191, 855

Ellison, D. C., Slane, P. \& Gaensler, B. M. 2001, ApJ, 563, 191

Jones, F. C. \& Ellison, D. C. 1991, Space Science Reviews, 58, 259

Lazendic, J. S., Slane, P. O., Gaensler, B. M., Reynolds, S. P.,

Plucinsky, P. P., \& Hughes, J. P. 2004, ApJ, 602, 271
Pelletier, G. and Lemoine, M. \& Marcowith, A. 2006, A\&A, 453, 181

Pohl, M., Yan, H. \& Lazarian, A., 2005, ApJ, 626, L101

Porter, T. A., Moskalenko, I. V. \& Strong, A. W., 2006, ApJ, 648, L29

Reynolds, S. P. \& Ellison, D. C., 1992, ApJ, 399, L75

Uchiyama, Y. et al. 2007, Nature, 449, 576

Vink, J. \& Laming, J. M., 2003, ApJ, 584, 758

Vladimirov, A. and Ellison, D. C. and Bykov, A. 2006, ApJ, 652, 1246

Völk, H. J., Berezhko, E. G., \& Ksenofontov, L. T., 2005, A\&A, 433,229

Warren, J. S., Hughes, J. P., Badenes, C., Ghavamian, P., McKee, C. F., Moffett, D., Plucinsky, P. P., Rakowski, C., Reynoso, E., \& Slane, P., 2005, ApJ, 634, 376 